\title{
REAL AND COMPLEX SUPERSOLVABLE LINE ARRANGEMENTS IN THE PROJECTIVE PLANE
}

\author{
KRISHNA HANUMANTHU AND BRIAN HARBOURNE
}

\begin{abstract}
We study supersolvable line arrangements in $\mathbb{P}^{2}$ over the reals and over the complex numbers, as the first step toward a combinatorial classification. Our main results show that a nontrivial (i.e., not a pencil or near pencil) complex line arrangement cannot have more than 4 modular points, and if all of the crossing points of a complex line arrangement have multiplicity 3 or 4 , then the arrangement must have 0 modular points (i.e., it cannot be supersolvable). This provides at least a little evidence for our conjecture that every nontrivial complex supersolvable line arrangement has at least one point of multiplicity 2, which in turn is a step toward the much stronger conjecture of Anzis and Tohăneanu that every nontrivial complex supersolvable line arrangement with $s$ lines has at least $s / 2$ points of multiplicity 2 .
\end{abstract}

\section{INTRODUCTION}

Line arrangements have provided useful insight in studying a range of recent problems in algebraic geometry. They have played a fundamental role in studying the containment problem (see [8, 7]), for the bounded negativity problem and H-constants [2], and for unexpected curves [4, 5]. The supersolvable arrangements are a particularly tractable subclass of line arrangements which have played a role in the study of unexpected curves [4, 5]. Understanding them better should make them even more useful. Thus the goal of the present paper is to pin down as much as currently possible properties of real and complex supersolvable line arrangements.

A line arrangement is simply a finite set of $s>1$ distinct lines $\mathcal{L}=\left\{L_{1}, \ldots, L_{s}\right\}$ in the projective plane. A modular point for $\mathcal{L}$ is a crossing point $p$ (i.e., a point where two (or more) of the lines meet), with the additional property that whenever $q$ is any other crossing point, then the line through $p$ and $q$ is $L_{i}$ for some $i$. Then we say $\mathcal{L}$ is supersolvable if it has a modular point (see Figure 1).

If the $s$ lines of $\mathcal{L}$ are concurrent (i.e., all meet at a point), then $\mathcal{L}$ is supersolvable. Such an arrangement is called a pencil. If $\mathcal{L}$ consists of $s$ lines, exactly $s-1$ of which are concurrent, it is called a near pencil; near pencils are also supersolvable, since every crossing point for a near pencil is modular. Removing any line, different from the line through two white dots, from the arrangement shown in Figure 1 results in a near pencil.

We refer to the number of lines of an arrangement containing a point as the multiplicity of the point. So crossing points always have multiplicity at least 2 . The modular points in

Date: July 15, 2019.

The first author was partially supported by a grant from Infosys Foundation and by DST SERB MATRICS grant MTR/2017/000243. The second author was partially supported by Simons Foundation grant \#524858. We also thank Ş. Tohăneanu for some helpful comments. 


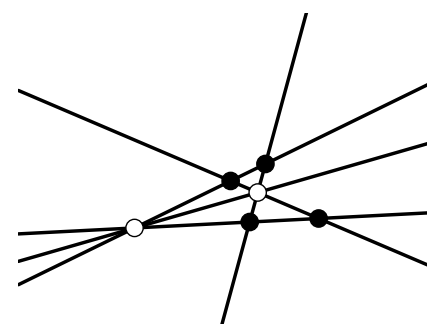

Figure 1. A supersolvable line arrangement with 2 modular points (shown as white dots).

Figure 1 have multiplicity 3, while the other crossing points in the figure have multiplicity 2 . For $k \geq 2$, we will use $t_{k}$ to denote the number of crossing points of multiplicity $k$.

For example, a pencil of $s$ lines has a unique modular point (indeed, a unique crossing point) and it has multiplicity $s$, so $t_{s}=1$ and otherwise $t_{k}=0$. A near pencil of $s$ lines has $s$ modular points; when $s>3, s-1$ of the $s$ modular points have multiplicity 2 $\left(\right.$ so $\left.t_{2}=s-1\right)$ and one has multiplicity $s-1\left(\right.$ so $\left.t_{s-1}=1\right)$, while if $s=3$ all the three modular points have multiplicity 2 (so $t_{2}=3$ ). We will refer to pencils and near pencils as trivial.

It is an open problem to determine which $t$ vectors $\left(t_{2}, \ldots, t_{s}\right)$ can arise for real or complex line arrangements, even for supersolvable line arrangements. It is even an open problem to classify all complex line arrangements with $t_{2}=0$, even for the supersolvable case. It is known that no nontrivial real line arrangement can have $t_{2}=0$. Three nontrivial kinds of complex line arrangements are known with $t_{2}=0$ but there is no proof that there are no others. No nontrivial supersolvable complex line arrangements are known with $t_{2}=0$, but again no proof is known that there are none even though it is expected that $t_{2}$ is large. (In [1] it is conjectured that a nontrivial complex supersolvable arrangement of $s$ lines has $t_{2} \geq s / 2$.)

We will address these questions for real and for complex supersolvable line arrangements. Our main results are Theorem 6, which shows that a nontrivial complex line arrangement cannot have more than 4 modular points, and Theorem [17, which shows that if all of the crossing points of a complex line arrangement have multiplicity 3 or 4, then the arrangement must have 0 modular points (i.e., it cannot be supersolvable). This provides at least a little evidence for our Conjecture 11 that every nontrivial complex supersolvable line arrangement has at least one crossing point of multiplicity 2, and also supports Conjecture 12 [1] that there are in fact at least $s / 2$ crossing points of multiplicity 2, where $s$ is the number of lines in the arrangement.

The structure of the paper is as follows. In Section 2 we recall facts we will use later. In Section 3 we study the classification of supersolvable real and complex line arrangements, and prove Theorem 6. In Section 4 we consider various conjectures related to the occurrence of points of multiplicity 2 on real and complex line arrangements (such as Conjectures 11 and 12), and we prove Theorem [17. Finally, in Section [5, we discuss the application of supersolvable line arrangements to the occurrence of unexpected plane curves, and raise the question of whether all which can occur are already known. 


\section{Preliminaries}

Let $\mathcal{L}=\left\{L_{1}, \ldots, L_{s}\right\}$ be a line arrangement in the projective plane over an arbitrary field $K$. In this section we include some well-known results that we use in this paper.

Recall that, for every $k \geq 2, t_{k}$ denotes the number of crossing points of multiplicity $k$. Let $n$ denote the number of all crossing points. Let $m$ be the largest integer $k$ such that $t_{k}>0$.

First we have the following combinatorial identity which holds for any field $K$.

$$
\left(\begin{array}{l}
s \\
2
\end{array}\right)=\sum_{k \geq 2}\left(\begin{array}{l}
k \\
2
\end{array}\right) t_{k} .
$$

If $K=\mathbb{C}$ and $\mathcal{L}$ is nontrivial, we have the following inequality due to Hirzebruch [9].

$$
t_{2}+\frac{3}{4} t_{3} \geq s+\sum_{k>4} t_{k}(k-4)
$$

If $K=\mathbb{R}$ and $\mathcal{L}$ is not a pencil, we have the following inequality due to Melchior [11].

$$
t_{2} \geq 3+\sum_{k \geq 3}(k-3) t_{k}
$$

When $\operatorname{char}(K)=0$ and $\mathcal{L}$ is supersolvable, we have the following inequality proved in [1, Proposition 3.1].

$$
t_{2} \geq 2 n-m(s-m)-2 .
$$

The following result is [12, Lemma 2.1]. For the reader's convenience we include a proof.

Lemma 1. Let $\mathcal{L}$ be a supersolvable line arrangement (over any field $K$ ) with a modular point $p$ of multiplicity $m$. If $q$ is a crossing point of multiplicity $n \geq m$, then $q$ is also modular.

Proof. In addition to the line $L=L_{p 1}=L_{q 1}$ through $p$ and $q, \mathcal{L}$ contains $m-1$ lines through $p$ (denote them by $L_{p 2}, \ldots, L_{p m}$ ) and $n-1$ lines through $q$ (denote them by $\left.L_{q 2}, \ldots, L_{q n}\right)$. Let $r_{i j}$ be the point where $L_{p i}$ intersects $L_{q j}$. Suppose $A$ and $B$ are any two distinct lines in $\mathcal{L}$. Let $r$ be the point where $A$ and $B$ meet. We must show $r$ is on a line in $\mathcal{L}$ through $q$. If either $A$ or $B$ contain $q$, then $r$ is on a line in $\mathcal{L}$ through $q$, so assume neither $A$ nor $B$ contains $q$.

First say $n>m$. Let $a_{j}$ be the point where $A$ and $L_{q j}$ meet. Since $q \neq a_{j}$, we get $n-1$ distinct points $a_{j}$, each of which is on some line $L_{p i_{j}}$ since $p$ is modular. But there are only $m-1<n-1$ lines $L_{p i}$, so we must have $i_{j}=i_{j^{\prime}}$ for some $j \neq j^{\prime}$, and hence $A=L_{p i_{j}}=L_{p i_{j^{\prime}}}$, so $p \in A$. Likewise $p \in B$, so $r=p \in L$ is on a line in $\mathcal{L}$ through $q$. (This also shows that $n>m$ implies that every line in $\mathcal{L}$ contains either $p$ or $q$; i.e., the lines in $\mathcal{L}$ are the $m+n-1$ lines through $p$ and $q$.)

Now say $n=m$. If both $A$ and $B$ contain $p$, then $r=p \in L$ is on a line in $\mathcal{L}$ through $q$. So assume either $A$ or $B$ does not contain $p$; say $p \notin A$. But $p$ is modular, so the point $r$ where $A$ and $B$ meet is on $L_{p i^{\prime}}$ for some $i^{\prime}$. Again, let $a_{j}$ be the point where $A$ and $L_{q j}$ meet. Since $q \neq a_{j}$, we get $n-1$ distinct points $a_{j}$, each of which is on some line $L_{p i_{j}}$ since $p$ is modular. If $i_{j}=i_{j^{\prime}}$ for some $j \neq j^{\prime}$, then $A=L_{p i_{j}}=L_{p i_{j^{\prime}}}$, so $p \in A$ 
contrary to assumption. Hence $i_{j} \neq i_{j^{\prime}}$ whenever $j \neq j^{\prime}$, the $n-1=m-1$ values of $j>1$ map under $j \mapsto i_{j}$ to all $m-1=n-1$ values of $i>1$, hence for some $j^{\prime}$ we have $i^{\prime}=i_{j^{\prime}}$, so $A$ meets $L_{p i^{\prime}}$ at $a_{j^{\prime}}=r_{i_{j^{\prime}} j^{\prime}}=r_{i^{\prime} j^{\prime}} \in L_{p i^{\prime}}$. But $A$ meets $L_{p i^{\prime}}$ at $r \in L_{p i^{\prime}}$, so $r=a_{j^{\prime}} \in L_{q j^{\prime}}$, so $r$ is on a line in $\mathcal{L}$ through $q$. Thus $q$ is modular.

\section{Classifying Supersolvable line ARRAngements}

\subsection{Supersolvable line arrangements with modular points of multiplicity 2 .}

We first classify all line arrangements, over any field $K$, having one or more modular points of multiplicity 2, or two (or more) modular points, not all of the same multiplicity. Thus, after this section, we may assume all modular points have the same multiplicity, which is at least 3 .

As a corollary of the proof of Lemma 1, we have the following result, which classifies line arrangements where at least two multiplicities occur as multiplicities of modular points.

Corollary 2. Let $\mathcal{L}$ be a supersolvable line arrangement (over any field $K$ ) with a modular point $p$ of multiplicity $m$. If $q$ is a crossing point of multiplicity $n>m$, then $\mathcal{L}$ consists exactly of the $m$ lines through $p$ and the $n$ lines through $q$ (hence $m+n-1$ lines altogether). If $m=2$, then every crossing point is modular and $\mathcal{L}$ is a near pencil. If $m>2$, then the only modular points are $p$ and $q$.

Proof. We saw in the proof of Lemma 1 that the lines in $\mathcal{L}$ are the $m+n-1$ lines through $p$ and $q$. If $m=2$, the only lines are the $m$ lines through $q$ (one of which goes through $p$ ) and the remaining line through $p$, hence $\mathcal{L}$ is a near pencil. If $m>2$, there are $(n-1)(m-1)$ crossing points of multiplicity 2 , but a point of multiplicity 2 on one line through $q$ is connected to at most one point of multiplicity 2 on any other line through $q$, and hence no point of multiplicity 2 is modular. I.e., the only modular crossing points are $p$ and $q$.

Proposition 3. Let $\mathcal{L}$ be a line arrangement (over any field) having one or more modular points, exactly one of which has multiplicity 2 (call this point $p$ ). Then $\mathcal{L}$ is the pencil consisting of the two lines through $p$.

Proof. If $\mathcal{L}$ had a crossing point of multiplicity $n>m=2$, then by Corollary 2, $\mathcal{L}$ is a near pencil, and thus would have $n$ points of multiplicity 2 . Thus $\mathcal{L}$ has exactly one crossing point, and it has multiplicity 2 , so $\mathcal{L}$ is the pencil consisting of the two lines through $p$.

Proposition 4. Let $\mathcal{L}$ be a line arrangement (over any field) having two or more modular points, at least two of which have multiplicity 2. Then $\mathcal{L}$ is a near pencil.

Proof. Let $p$ and $q$ be modular points of multiplicity 2. Since $\mathcal{L}$ is supersolvable, given a crossing point other than $p$, the line from $p$ to that point is in $\mathcal{L}$. But $p$ has multiplicity 2 , so every crossing point must be on one or the other of the two lines through $p$. Likewise, every crossing point must be on one or the other of the two lines through $q$.

Let $L$ be the line through both $p$ and $q$; thus $L \in \mathcal{L}$. Let $L_{p}$ be the other line in $\mathcal{L}$ through $p$ and let $L_{q}$ be the other line in $\mathcal{L}$ through $q$. Let $r$ be the point where $L_{p}$ and $L_{q}$ meet. Thus any crossing point not on $L$ must be on both $L_{p}$ and $L_{q}$; i.e., $r$ is the 
only crossing point not on $L$. Thus every line in $\mathcal{L}$ other than $L$ must contain $r$, so $\mathcal{L}$ is a near pencil.

3.2. Homogeneous supersolvable line arrangements (mostly for $\operatorname{char}(K)=0$ ). By our foregoing results, we see that it remains to understand supersolvable line arrangements such that all modular points have the same multiplicity $m$ (we say such a supersolvable line arrangement is homogeneous or $m$-homogeneous) with $m \geq 3$. It follows from Lemma 1 that $t_{k}=0$ for $k>m$. For an $m$-homogeneous supersolvable line arrangement $\mathcal{L}$, we denote $m$ by $m_{\mathcal{L}}$.

3.2.1. The values of $t_{m_{\mathcal{L}}}$ that arise for $\operatorname{char}(K)=0$. When $K$ is algebraically closed but of finite characteristic, there is no bound to the number of modular points a supersolvable line arrangement can have. (Just take all lines defined over a finite field $F$ of $a$ elements. Then the arrangement has $a^{2}+a+1$ lines and the same number of crossing points; all are modular and all have multiplicity $a+1$.) In characteristic 0 things are very different, as we show in Theorem 6 .

To prove the theorem, we will use the following result.

Proposition 5. For an $m$-homogeneous supersolvable complex line arrangement $\mathcal{L}$ with $m=m_{\mathcal{L}} \geq 3$, no three modular points are collinear.

Proof. Suppose that $p, q$ and $r$ are collinear modular points. Then the line $L$ that contains them is in $\mathcal{L}$. Moreover, $\mathcal{L}$ contains $m-1$ additional lines through each of $p, q$ and $r$. Denote the union of these $m-1$ lines through $p$ by $C_{p}$. Similarly, we have $C_{q}$ and $C_{r}$. The intersection of the curves $C_{p}$ and $C_{q}$ is a complete intersection of $(m-1)^{2}$ points, which are also contained in $C_{r}$. Since the curves all have degree $m-1$, we see that $C_{r}$ is in the pencil defined by $C_{p}$ and $C_{q}$. I.e., the forms $F_{p}, F_{q}$ and $F_{r}$ defining the curves are such that $F_{r}$ is a linear combination of $C_{p}$ and $C_{q}$. we can choose coordinates such that $L$ is $x=0, p$ is $x=y=0, q$ is $x=z=0$ and $r$ is $y=z=1$. In terms of these coordinates, the restrictions of $F_{p}, F_{q}, F_{r}$ to $L$ are $y^{m-1}, z^{m-1}$ and $a y^{m-1}+b z^{m-1}=(y-z)^{m-1}$ for some nonzero constants $a$ and $b$. Setting $z=1$, we thus see that $a y^{m-1}+b=(y-1)^{m-1}$, so $a y^{m-1}+b$ has a multiple root at $y=1$. This contradicts the fact that the derivative $a(m-1) y^{m-2}+b$ is not 0 at $y=1$.

Theorem 6. For an $m$-homogeneous supersolvable complex line arrangement $\mathcal{L}$ with $m=m_{\mathcal{L}} \geq 3$, we have $1 \leq t_{m} \leq 4$.

Proof. First we show that $t_{m}<7$. Suppose $t_{m} \geq 7$ for some $m \geq 3$. Each non-modular crossing point is connected by a line to each of the $t_{m} \geq 7$ modular points. Since at most two modular points can lie on any line by Proposition 5, we see that each crossing point must have multiplicity at least 4 . Also, each modular point has multiplicity $m \geq 6$ since each one connects to each of the others. Thus $t_{2}=t_{3}=0$, but this is impossible by Inequality (2.2).

Next we show that $t_{m}<6$. Suppose $\mathcal{L}$ has $t_{m}=6$. It is enough to show $t_{m}<6$ under the assumption that every line in $\mathcal{L}$ contains a modular point. (This is because if we let $\mathcal{L}^{\prime}$ be the line arrangement obtained from $\mathcal{L}$ by deleting all lines not through a modular point, $\mathcal{L}^{\prime}$ still has $t_{m_{\mathcal{L}^{\prime}}}=6$.) Since every modular point is on a line in $\mathcal{L}$ through another modular point, we have $m \geq 5$. Every crossing point $q$ of $\mathcal{L}$ also connects to 
every modular point so has multiplicity at least 3 (since a line can go through at most 2 modular points), with multiplicity exactly 3 if and only if $q$ is 3 lines through pairs of modular points.

There are $2\left(\begin{array}{l}6 \\ 4\end{array}\right)=30$ possible locations for crossing points of multiplicity 3 , hence $t_{3} \leq 30$. To see this note that there are $\left(\begin{array}{l}6 \\ 4\end{array}\right)$ ways to pick 4 of the 6 modular points. There are 3 reducible conics through these 4 points. The singular points of these three conics are crossing points where two lines through disjoint pairs chosen from the 4 points intersect. In order to get a point $q$ of multiplicity 3 , the line $H$ through the remaining 2 points of the 6 modular points must contain $q$. This might not happen for any of the three singular points, but it can be simultaneously true for at most two of the three singular points, since at most two of the singular points can be on the line $H$ (this is merely because the three singular points cannot be collinear in characteristic 0). Thus we get at most $2\left(\begin{array}{l}6 \\ 4\end{array}\right)=30$ possible locations for crossing points of multiplicity 3 .

Now apply Inequality (2.2), using the fact that our assumption (that every line in $\mathcal{L}$ contains a modular point) implies that $\mathcal{L}$ has $(m-5) 6+\left(\begin{array}{l}6 \\ 2\end{array}\right)$ lines:

$$
22.5=\frac{3}{4} 30 \geq \frac{3}{4} t_{3} \geq\left((m-5) 6+\left(\begin{array}{l}
6 \\
2
\end{array}\right)\right)+(m-4) 6 .
$$

For $m \geq 6$ this is $22.5 \geq 12 m-39 \geq 33$, thus the only possibility for $t_{m}=6$ is $m=5$. For $m=5$ we see $\mathcal{L}$ has $\left(\begin{array}{l}6 \\ 2\end{array}\right)=15$ lines and every crossing point has multiplicity at least 3 and at most 5, so from Equation (2.1) we get:

$$
105=\left(\begin{array}{c}
15 \\
2
\end{array}\right)=3 t_{3}+6 t_{4}+10 t_{5}=3 t_{3}+6 t_{4}+60
$$

so $15=t_{3}+2 t_{4}$, hence $t_{3} \leq 15$. Inequality (2.2) now gives $(3 / 4) 15 \geq 15+6$, which is false.

Finally, we show that $t_{m}<5$. So assume $t_{m}=5$. Arguing as before, we may assume that every line in $\mathcal{L}$ contains a modular point. We still have that al non-modular points have multiplicity at least 3 , and the 5 modular points have multiplicity $m \geq 4$. Each choice of 4 of the 5 modular points gives 3 possible locations for a triple point, hence $t_{3} \leq 3(5)=15$. Thus Inequality $(2.2)$ gives $11.25=(3 / 4) 15 \geq\left(\left(\begin{array}{l}5 \\ 2\end{array}\right)+(m-4) 5\right)+(m-$ $4) 5=10 m-30$, which is impossible for $m \geq 5$. For $m=4$ we see $\mathcal{L}$ has $\left(\begin{array}{l}5 \\ 2\end{array}\right)=10$ lines and every crossing point has multiplicity at at least 3 and at most 4 , so from Equation (2.1) we get:

$$
45=\left(\begin{array}{c}
10 \\
2
\end{array}\right)=3 t_{3}+6 t_{4}=3 t_{3}+30
$$

so $5=t_{3}$. Inequality (2.2) now gives $(3 / 4) 5 \geq 10$, which is false.

Example 7. For $m$-homogeneous supersolvable line arrangements over both the complex numbers and the reals, all four cases $1 \leq t_{m_{\mathcal{L}}} \leq 4$ arise. It is easy to obtain examples with exactly one modular point; see Section 3.3. (However, the fact that there are many examples makes it hard to classify them!) It is also easy to obtain examples with exactly two modular points; see Corollary 2, For exactly three modular points, consider the line arrangement defined by the linear factors of $x y z\left(x^{n}-y^{n}\right)\left(x^{n}-z^{n}\right)\left(y^{n}-z^{n}\right)$ for $n \geq 2$. The coordinate vertices are the modular points, and have multiplicity $n+2$. For $n=2$ 
the arrangement is real (see the arrangement of 9 lines shown in Figure 3); for $n>2$ it is complex but not real. Taking $n=1$, so $x y z(x-y)(x-z)(y-z)$, gives the only example we know over the complexes or reals with exactly four modular points; see Case 2 of Figure 2. (We thank Ş. Tohăneanu for pointing out that a line arrangement equivalent to the one defined by the linear factors of $x y z\left(x^{n}-y^{n}\right)\left(x^{n}-z^{n}\right)\left(y^{n}-z^{n}\right)$ for $n=2$ arose as an example in section 3.1.1 of [1], to show that a certain bound on the number of crossing points was sharp. For the line arrangements given by $x y z\left(x^{n}-y^{n}\right)\left(x^{n}-z^{n}\right)\left(y^{n}-z^{n}\right)$ the bound is $s \leq d^{2}+d+1$, where $s=n^{2}+3 n+3$ is the number of crossing points and $d=m_{\mathcal{L}}-1=n+1$. Thus we see that $s=d^{2}+d+1$, so this bound is in fact sharp for all values of $n$.)

3.2.2. Classifying $m$-homogeneous $\mathcal{L}$ for $t_{m}>1$ and $m=3$. Consider the case of a line arrangement $\mathcal{L}$ with two or more modular points of multiplicity $m \geq 3$. Since we have at least two modular points, we pick two and call them $p$ and $q$.

First say $m=3$. We will show that there are three cases, shown in Figure 2; $\mathcal{L}$ has either 5, 6 or 7 lines, and either 2, 4 or 7 modular points, respectively. The case of 7 lines occurs only in characteristic 2 . The other cases occur for any field.

Clearly, $\mathcal{L}$ has at least 5 lines: the line $L$ defined by $p$ and $q$, and in addition lines $p \in L_{p i}$ and $q \in L_{q i}$, for $i=1,2$. No other lines in $\mathcal{L}$ (if any) can contain $p$ or $q$. Let $r_{1}$ be where $L_{p 1}$ and $L_{q 1}$ meet and let $r_{2}$ be where $L_{p 2}$ and $L_{q 2}$ meet. And let $s_{1}$ be where $L_{p 1}$ and $L_{q 2}$ meet and let $s_{2}$ be where $L_{p 2}$ and $L_{q 1}$ meet. Any other line in $\mathcal{L}$ must intersect the lines $L_{p i}$ and $L_{q i}$ only at $r_{1}, r_{2}, s_{1}$, or $s_{2}$.

One possibility is that $\mathcal{L}$ has only the five lines mentioned above. Alternatively, assume $\mathcal{L}$ has another line, $A$. Of the six pairs two points chosen from the four points $r_{1}, r_{2}, s_{1}$ and $s_{2}, A$ must contain either $r_{1}$ and $r_{2}$ or $s_{1}$ and $s_{2}$ (A cannot contain $r_{1}$ and $s_{1}$, for example, because that line is $\left.L_{p 1}\right)$. Up to relabeling, the case $r_{1}$ and $r_{2}$ is the same as $s_{1}$ and $s_{2}$, so say $A$ contains $r_{1}$ and $r_{2}$. Up to projective equivalence, we may assume that $p=(0,0,1), q=(0,1,0), r_{1}=(1,0,0)$ and $r_{2}=(1,1,1)$, in which case $s_{1}=(1,0,1)$ and $s_{2}=(1,1,0)$. So a second possibility is that $\mathcal{L}$ has six lines, with $A$ being the sixth line. Note that in this case that $\mathcal{L}$ has 4 modular points: the points $p, q, r_{1}$ and $r_{2}$ are modular, and all have multiplicity 3 . The only option for $\mathcal{L}$ to contain an additional line is for the additional line (call it $B$ ) to be the line through $s_{1}$ and $s_{2}$. But $A$ is $y-z=0$ and $B$ is $x-y-z=0$, so $A$ and $B$ intersect at the point $(2,1,1)$. When the ground field does not have characteristic 2 , this is not on any of the three lines through $p$ (or on any of the three lines through $q$ ), hence including $B$ would make $\mathcal{L}$ not be supersolvable. Thus when the characteristic is not $2, \mathcal{L}$ must either have 5 or 6 lines, and be Case 1 or Case 2 shown in Figure 2. If the characteristic is 2 , the point $(2,1,1)$ is on the line through $p$ and $q$, in which case $\mathcal{L}$ consists of the 7 lines of the Fano plane, there are 7 crossing points, all are modular and have multiplicity 3.

3.2.3. Classifying $m$-homogeneous $\mathcal{L}$ over the reals for $t_{m}>1$ and $m>3$. Now we consider the case $m \geq 4$ for real line arrangements. So, in addition to the line $L$ through $p$ and $q$, there are $m-1$ lines through $p$ and $m-1$ lines through $q$. These lines form a complete intersection (i.e., a grid) of $(m-1)^{2}$ crossing points. The only other crossing points for these $2 m-1$ lines are $p$ and $q$. Certainly $\mathcal{L}$ could consist of only these $2 m-1$ 

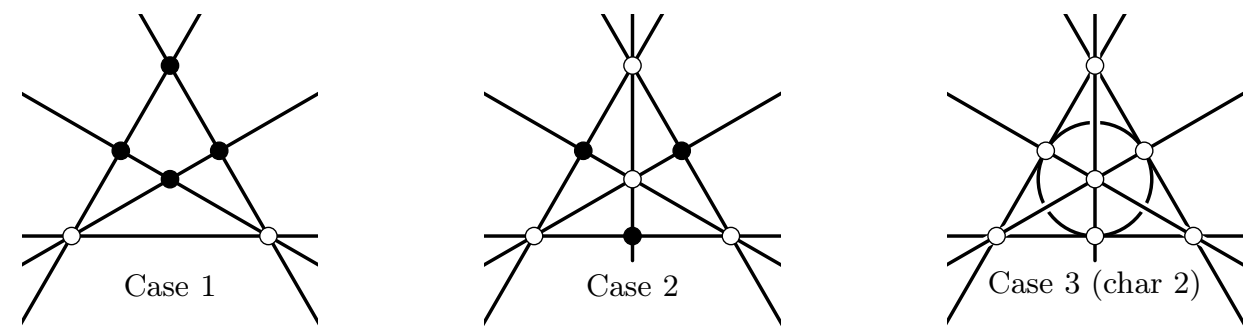

Figure 2. Classification of supersolvable line arrangements with 2 or more modular points (shown as white dots), all of multiplicity $m=3$.

lines, in which case $p$ and $q$ are the only modular points and we have $t_{k}=0$ except for $t_{m}=2$ and $t_{2}=(m-1)^{2}$.

The question now is what additional lines can be added to these $2 m-1$ while maintaining supersolvability. To answer this, let's choose coordinates so that $p$ becomes $(0,1,0)$ and $q$ becomes $(1,0,0)$. Thus the line through $p$ and $q$ is now the line at infinity, and the $m-1$ other lines through $p$ are parallel to the $x=0$ axis, and the $m-1$ other lines through $q$ are parallel to the $y=0$ axis.

Any additional line must avoid $p$ and $q$, and must intersect the $m-1$ vertical lines only at points where they meet the $m-1$ horizontal lines. By inspection we can see that this can happen in exactly to ways. First is that the four corners of the grid form a rectangle and the $i$ th vertical line (counting from the left) meets the $i$ th horizontal line (counting up from the bottom) meet on the anti-diagonal of the rectangle (in which case the anti-diagonal can be added to $\mathcal{L}$ ). The second way is that the four corners of the grid form a rectangle (as before) and the $i$ th vertical line (counting from the left) meets the $i$ th horizontal line (counting DOWN this time from the top) meet on the main diagonal of the rectangle (in which case the main diagonal can be added to $\mathcal{L}$ ). In case both cases hold, both diagonals can be added if and only if $m$ is even.

Thus there are three cases: $\mathcal{L}$ has $2 m-1$ lines and we have $t_{m}=2$ and $t_{2}=(m-1)^{2}$ but only two modular points, namely $p$ and $q ; \mathcal{L}$ has $2 m$ lines where the additional line is one of the two major diagonals (assuming the lines are spaced correctly) and we have still have only two modular points $(p$ and $q)$, with $t_{m}=2, t_{2}=(m-1)^{2}-(m-1)+1$; or $\mathcal{L}$ has $2 m+1$ lines where the additional lines are the two major diagonals (assuming the lines are spaced correctly and $m$ is even), in which case either $m=4$ and we have $t_{m}=3, t_{2}=6, t_{3}=4$ and there are three modular points $(p, q$ and the center of the rectangle), or $m>4$ and we have $t_{m}=2, t_{2}=(m-1)^{2}-(2 m-1)+2, t_{3}=2 m-4$ and $t_{4}=1$ and there are only two modular points $(p$ and $q)$.

Thus we have a complete classification of real supersolvable line arrangements when there is more than one modular point of multiplicity at least 3 .

3.2.4. Classifying $m$-homogeneous $\mathcal{L}$ over the complexes for $t_{m}>2$ and $m>3$. Now we consider the case $m \geq 4$ for complex line arrangements with at least 3 modular points. By Theorem 6, the number of modular points cannot be more than 4 .

We begin with the case of exactly $t_{m}=3$ modular points. If $\mathcal{L}$ has a line that does not contain a modular point, deleting it gives an arrangement which is still supersolvable, so we first assume every line in $\mathcal{L}$ goes through a modular point. 
After a change of coordinates, we may assume that the three modular points, $p, q, r$, are the coordinate vertices of $\mathbb{P}^{2}$, so say $p=(0,0,1), q=(0,1,0), r=(1,0,0)$. In addition to the three coordinate axes, $\mathcal{L}$ must contain $m-2$ lines through each of $p$, $q$ and $r$. Let $F_{p}$ be the form defining the union of these $m-2$ lines through $p$, other than the coordinate axes. Note that $F_{p}$ is a form of degree $m-2$ and involves only the variables $x$ and $y$, hence is $F_{p}(x, y)$. Likewise we have $F_{q}(x, z)$ and $F_{r}(y, z)$ for $q$ and $r$. Since the coordinate axes are not among the lines defined by $F_{p}, F_{q}$ or $F_{r}$, we see that none of these forms is divisible by a variable.

The crossing points for the lines from $F_{p}$ and the lines from $F_{q}$ form a complete intersection of $(m-2)^{2}$ points on which $F_{r}$ also vanishes, so $F_{r}=a F_{p}+b F_{q}$ for some scalars $a$ and $b$. The only term that $F_{p}$ and $F_{q}$ can have in common is $x^{m-2}$. Thus in order that all terms involving $x$ cancel in $a F_{p}+b F_{q}$ so that $F_{r}$ involves only $y$ and $z$, we see that $x^{m-2}$ is the only term in either $F_{p}$ or $F_{q}$ involving $x$. Thus (after dividing by the coefficient of $x^{m-2}$ in each case) we have $F_{p}=x^{m-2}-\alpha y^{m-2}$ and $F_{q}=x^{m-2}-\beta z^{m-2}$. By absorbing the $\alpha$ into $y$ and the $\beta$ into $z$, we get $F_{p}=x^{m-2}-y^{m-2}$ and $F_{q}=x^{m-2}-z^{m-2}$, so $F_{r}=y^{m-2}-z^{m-2}$.

Thus if every line in $\mathcal{L}$ goes through one of the three modular points, then the lines in $\mathcal{L}$ correspond to the linear factors of $x y z\left(x^{m-2}-y^{m-2}\right)\left(x^{m-2}-z^{m-2}\right)\left(y^{m-2}-z^{m-2}\right)$. Now we check that no line not through $p, q$ or $r$ can be added to $\mathcal{L}$ while still preserving supersolvability. If such a line $L$ existed, it would need to intersect every line of $\mathcal{L}$ in a crossing point. In particular, $L$ must contain one of the $(m-2)^{2}$ intersection points of the lines from $F_{p}$ and the lines from $F_{q}$. Let $n:=m-2$. By an appropriate change of coordinates obtained by multiplying $x, y$ and $z$ by appropriate powers of an $n$th root of 1 , we may assume that $L$ contains $(1,1,1)$. Let $\epsilon=\cos (2 \pi / n)+\imath \sin (2 \pi / n)$ be a primitive $n$th root of 1 . The line $L$ must intersect $y-\epsilon z=0$ at a crossing point (hence at $\left(\epsilon^{i}, \epsilon, 1\right)$ for some $1 \leq i \leq n$ ) and also $y-\epsilon^{2} z=0$ at a crossing point (hence at $\left(\epsilon^{j}, \epsilon^{2}, 1\right)$ for some $1 \leq j \leq n)$. The question is whether $i$ and $j$ exist such that these points lie on a line through $(1,1,1)$ which does not go through $p, q$ or $r$.

The lines through $(1,1,1)$ are of the form $a(x-z)+b(y-z)=0$. For the line not to go through $p, q$ or $r$, we need $a b \neq 0$. Thus we can write the line as $c=(y-z) /(x-z)$ for some $c \neq 0$. For $\left(\epsilon^{i}, \epsilon, 1\right)$ and $\left(\epsilon^{j}, \epsilon^{2}, 1\right)$ both to lie on this line we must have

$$
\frac{\epsilon-1}{\epsilon^{i}-1}=\frac{\epsilon^{2}-1}{\epsilon^{j}-1}
$$

This simplifies to

$$
\epsilon^{i-1}(\epsilon+1)=\epsilon^{j-1}+1 .
$$

Thus the complex norms are equal; i.e., $|\epsilon+1|=\left|\epsilon^{j-1}+1\right|$. But if $\gamma=\cos (\theta)+\imath \sin (\theta)$, the norm $|\gamma+1|$ is a decreasing function of $\theta$ for $0 \leq \theta \leq \pi$, so the only possibilities for $|\epsilon+1|=\left|\epsilon^{j-1}+1\right|$ are $j=2$, n. If $j=2$, then $\epsilon^{i-1}(\epsilon+1)=\epsilon^{j-1}+1$ forces $i=1$, so the line through $\left(\epsilon^{i}, \epsilon, 1\right)$ and $\left(\epsilon^{j}, \epsilon^{2}, 1\right)$ then is $x-y=0$, which contains $p$. If $j=n$, then $\epsilon^{i-1}(\epsilon+1)=\epsilon^{j-1}+1=(1+\epsilon) / \epsilon$ forces $\epsilon^{i}=1$. and hence $i=n$, so the line is $x-z=0$, which contains $q$.

Thus the only possibility for 3 modular points of multiplicity $m>3$, is (up to choice of coordinates) for the line arrangement to be the lines defined by the linear factors of $x y z\left(x^{m-2}-y^{m-2}\right)\left(x^{m-2}-z^{m-2}\right)\left(y^{m-2}-z^{m-2}\right)$. 
Now suppose $\mathcal{L}$ has 4 modular points with $m>3$. We can, up to choice of coordinates, assume that the four points are $p, q, r, s$, where $p, q, r$ are as above, and $s=(1,1,1)$. If we delete any lines not through $p, q, r$, then the resulting arrangement must come from the linear factors of $x y z\left(x^{m-2}-y^{m-2}\right)\left(x^{m-2}-z^{m-2}\right)\left(y^{m-2}-z^{m-2}\right)$. To get $\mathcal{L}$, we must add back in lines through $s$ which intersect the lines coming from $x y z\left(x^{m-2}-y^{m-2}\right)\left(x^{m-2}-\right.$ $\left.z^{m-2}\right)\left(y^{m-2}-z^{m-2}\right)$ only at crossing points for the lines from $x y z\left(x^{m-2}-y^{m-2}\right)\left(x^{m-2}-\right.$ $\left.z^{m-2}\right)\left(y^{m-2}-z^{m-2}\right)$. But as we just saw there are no such lines. Thus $\mathcal{L}$ having 4 modular points with $m>3$ is impossible.

Thus, up to choice of coordinates, the only complex supersolvable line arrangement with 4 modular points is the one we found before; i.e., $x y z\left(x^{m-2}-y^{m-2}\right)\left(x^{m-2}-\right.$ $\left.z^{m-2}\right)\left(y^{m-2}-z^{m-2}\right)$ with $m=3$, displayed in Case 2 of Figure 2. And up to choice of coordinates the only complex supersolvable line arrangements with 3 modular points are given by the linear factors of $x y z$ when $m=2$, and by the linear factors of $x y z\left(x^{m-2}-y^{m-2}\right)\left(x^{m-2}-z^{m-2}\right)\left(y^{m-2}-z^{m-2}\right)$ for $m>3$.

We do not have a classification of complex supersolvable line arrangement with just 1 or 2 modular points. If for $m \geq 3$ you remove one or more of the linear factors of $y^{m-2}-z^{m-2}$ from the set of linear factors of $x y z\left(x^{m-2}-y^{m-2}\right)\left(x^{m-2}-z^{m-2}\right)\left(y^{m-2}-z^{m-2}\right)$, then we get examples of complex supersolvable line arrangement with just 2 modular points. Thus more examples occur over the complexes than over the reals, but it is not clear what the full range of possibilities is.

In any case, we have given a full classification over the complexes for supersolvable line arrangement with 3 or 4 modular points. We discuss the case of 1 modular point in the next section.

3.3. Having a single modular point. The case that there is a single modular point is the hardest to classify and we can give only partial results in this case.

We begin with a lemma.

Lemma 8. Let $\mathcal{L}$ be a line arrangement (not necessarily supersolvable, not necessarily over the reals). Let $m$ be the maximum of the multiplicities of the crossing points and let $n$ be the number of crossing points. If $n<2 m$, then $\mathcal{L}$ is either a pencil or near pencil.

Proof. Assume $\mathcal{L}$ is not a pencil or a near pencil. Let $p$ be a point of multiplicity $m$ and take lines $A$ and $B$ not through $p$. Then $A$ and the $m$ lines through $p$ give $m+1$ crossing points, and $B$ then gives at least another $m-1$ crossing points, for a total of at least $2 m$ crossing points.

We now consider the case of a line arrangement $\mathcal{L}$ with a single modular point, which we assume has multiplicity $m>2$; call it $p$. By [1] every other crossing point of $\mathcal{L}$ has multiplicity less than $p$ (because for a supersolvable line arrangement, all points of maximum multiplicity are modular). Assume $\mathcal{L}$ is not a pencil or a near pencil. Let $\mathcal{L}^{\prime}$ be the arrangement obtained from $\mathcal{L}$ by removing the $m$ lines through $p$. We can recover $\mathcal{L}$ by adding to $\mathcal{L}^{\prime}$ every line from $p$ to a crossing point of $\mathcal{L}^{\prime}$. What is difficult to know is how many lines get added, since one line through $p$ might contain more than one crossing point of $\mathcal{L}^{\prime}$. But we see that $t_{m}=1$ and $t_{k+1}=t_{k}^{\prime}$ for all $2<k<m$, where $t_{k}^{\prime}$ is the number of crossing points of $\mathcal{L}^{\prime}$ of multiplicity $k$. Even knowing how many lines 
are in $\mathcal{L}^{\prime}$ and the value of $t_{k}^{\prime}$ for every $k$, it's hard to say how many lines are in $\mathcal{L}$, or what the value of $t_{2}$ is, except in certain special situations.

Suppose, for example, we know that no two crossing points of $\mathcal{L}^{\prime}$ are on the same line through $p$. Since $\mathcal{L}^{\prime}$ has $t_{2}^{\prime}+\cdots+t_{m}^{\prime}$ crossing points and $\mathcal{L}^{\prime}$ has $s^{\prime}$ lines, where $\left(\begin{array}{l}s^{\prime} \\ 2\end{array}\right)=\sum_{k} t_{k}^{\prime}\left(\begin{array}{l}k \\ 2\end{array}\right)$ (see (2.1) $)$, we then know that $\mathcal{L}$ has $s=s^{\prime}+t_{2}^{\prime}+\cdots+t_{m}^{\prime}$ lines and then from $\left(\begin{array}{l}s \\ 2\end{array}\right)=\sum_{k} t_{k}\left(\begin{array}{l}k \\ 2\end{array}\right)$ we can determine $t_{2}$.

Alternatively, start with any line arrangement $\mathcal{L}^{\prime}$ (over any field) which is not a pencil or a near pencil. By Lemma $8, n^{\prime} \geq 2 m^{\prime}$, where $n^{\prime}$ is the number of crossing points of $\mathcal{L}^{\prime}$ and $m^{\prime}$ is the maximum of their multiplicities. For a general point $p$, no line through $p$ will contain more than one crossing point of $\mathcal{L}^{\prime}$. Now add to $\mathcal{L}^{\prime}$ each line from $p$ to a crossing point of $\mathcal{L}^{\prime}$ to get a larger line arrangement $\mathcal{L}$ of $s=n^{\prime}+s^{\prime}$ lines, where $s^{\prime}$ is the number of lines of $\mathcal{L}^{\prime}$. We also know that $t_{k+1}=t_{k}^{\prime}$ for all $k>2$, and we can determine $t_{2}$ from $\left(\begin{array}{l}s \\ 2\end{array}\right)=\sum_{k} t_{k}\left(\begin{array}{l}k \\ 2\end{array}\right)$. Moreover, $p$ is the unique modular point of $\mathcal{L}$. Note that $p$ has multiplicity $n^{\prime} \geq 2 m^{\prime}$ and the maximum multiplicity of any other crossing point of $\mathcal{L}$ is $m^{\prime}+1<2 m^{\prime}$. Thus if $\mathcal{L}$ has another modular point, it has multiplicity $d<n^{\prime}$, hence by our classification $\mathcal{L}$ has $d+n^{\prime}-1$ lines. But in fact $s^{\prime} \geq d+1$ since $\mathcal{L}^{\prime}$ is not a pencil or near pencil, and $\mathcal{L}$ has $s=s^{\prime}+n^{\prime}>d+1-n^{\prime}$ lines. Thus $\mathcal{L}$ has a unique modular point, namely $p$. Thus classifying line arrangements with a unique modular point, even when that point is general, comes down to classifying line arrangements in general.

3.4. Summary. The real supersolvable line arrangements having more than one modular point can be subsumed by one general construction. Take two points, $p$ and $q$, on a line $L$. Take $a_{p} \geq 0$ additional lines through $p$ and $a_{q} \geq 0$ additional lines through $q$. This gives a supersolvable line arrangement as long as $a_{p}+a_{q}>0$. In addition, if $a_{p}=a_{q} \geq 2$ and the obvious collinearity condition obtains, an additional line can be added in two possible ways (shown by the dashed and dashed-dotted lines in Figure 3 in the case of $a_{p}=a_{q}=3$ ). If both can be added separately and if $a_{p}=a_{q}$ is odd, both can be added simultaneously. These constructions cover all possible cases of real supersolvable line arrangements with 2 or more modular points.

The case of complex supersolvable line arrangements with more than two modular points are all given, up to choice of coordinates, by the linear factors of $x y z\left(x^{m-2}-\right.$ $\left.y^{m-2}\right)\left(x^{m-2}-z^{m-2}\right)\left(y^{m-2}-z^{m-2}\right)$ for $m \geq 3$.

\section{Points of multiplicity 2 in supersolvable line arrangements}

4.1. Questions and conjectures. By Inequality (2.3), every non-pencil real line arrangement has $t_{2} \geq 3$. More generally, there is the still open Dirac-Motzkin Conjecture [6]:

Conjecture 9. The inequality $t_{2} \geq\lfloor s / 2\rfloor$ holds for every non-pencil real line arrangement of s lines.

Things over $\mathbb{C}$ are more complicated. Four types of complex line arrangements with $t_{2}=0$ are currently known: pencils of 3 or more lines; the lines defined by the linear factors of $\left(x^{n}-y^{n}\right)\left(x^{n}-z^{n}\right)\left(y^{n}-z^{n}\right)$ for $n \geq 3$ (known as the Fermat arrangement, $\left.\mathcal{F}_{n}\right)$; an arrangement due to F. Klein [10] with 21 lines and $t_{k}=0$ except for $t_{3}=28$ and $t_{4}=21$; and an arrangement due to A. Wiman [13] with 45 lines and $t_{k}=0$ except for 


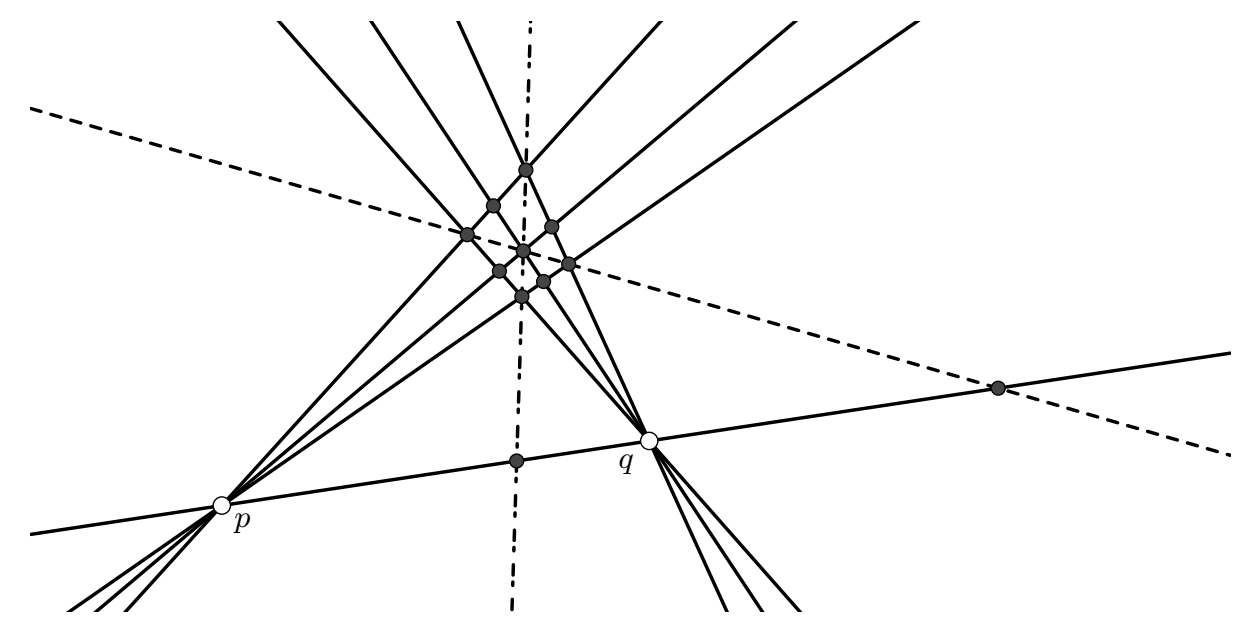

FiguRE 3. A supersolvable line arrangement with 2 modular points of equal multiplicity with possible added lines.

$t_{3}=120, t_{4}=45$ and $t_{5}=36$ (see [3] for more information about the Klein and Wiman arrangements).

We believe the following question is open.

Question 10. Are there any complex line arrangements with $t_{2}=0$ other than the four types listed above?

For the case of supersolvable line arrangements we pose the following conjecture.

Conjecture 11. Every nontrivial complex supersolvable line arrangement has $t_{2}>0$.

A much stronger conjecture was posed by [1].

Conjecture 12. Every non-pencil complex supersolvable line arrangement of s lines has $t_{2} \geq s / 2$.

We have found all complex supersolvable line arrangements with at least 3 modular points, and for these $t_{2} \geq s / 2$ holds. Thus if the conjecture is false, then it must fail for a line arrangement with either one or at most 2 modular points.

It is also interesting to ask:

Question 13. Which non-pencil complex line arrangements of s lines fail to satisfy $t_{2} \geq\lfloor s / 2\rfloor ?$

Of course, as noted above, there are non-pencil line arrangements with $t_{2}=0$, and for these $t_{2} \geq\lfloor s / 2\rfloor$ fails to hold. Also, by adding or deleting lines from such line arrangements one can sometimes get additional examples. For example, the line arrangement $\mathcal{L}$ with $s=3 n$ lines defined by the linear factors of $\left(x^{n}-y^{n}\right)\left(x^{n}-z^{n}\right)\left(y^{n}-z^{n}\right)$ has $t_{2}=0$; by adding the line $x=0$, we get a line arrangement $\mathcal{L}^{\prime}$ with $s=3 n+1$ and $t_{2}=n$, so $t_{2} \geq\lfloor s / 2\rfloor$ still fails. For another example, each line of the Klein arrangement of 21 lines contains four crossing points of multiplicity 4 and four of multiplicity 3. By removing one line we thus get an arrangement of $s=20$ lines with $t_{4}=17, t_{3}=28$ and $t_{2}=4$, so here too $t_{2} \geq\lfloor s / 2\rfloor$ fails. But this leaves the question: are there any examples where 
$t_{2} \geq\lfloor s / 2\rfloor$ fails to hold which do not come in this way from the known examples with $t_{2}=0$ ?

If $\mathcal{L}$ is defined over $\mathbb{R},[1$ proves Conjecture 12 over the reals (see [1, Theorem 2.4]). A key step in their proof is [1, Lemma 2.2], a version of which we now state. For the convenience of the reader we include a slightly simplified version of the proof from [1].

Lemma 14. Let $p$ be a modular point of some multiplicity $m$ in a non-pencil real supersolvable line arrangement $\mathcal{L}$ containing s lines. Then every line in $\mathcal{L}$ not containing $p$ contains a crossing point of multiplicity 2.

Proof. At left in Figure 4 we see the $m$ lines $\left(L_{1}, \ldots, L_{m}\right.$ enumerated from bottom to top) through $p$ and some line $L$ not through $p$. To these we've added a dotted line below $L_{1}$, and a dashed line above $L_{m}$. After a change of coordinates, the dotted line becomes $y=0$, the dashed line becomes the line $z=0$ at infinity, $L$ becomes $x=0$ and $p$ becomes the point $(1,0,0)$. Thus in the affine plane as shown at right in Figure 4 , the lines $L_{i}$ become horizontal lines and $L$ becomes vertical.

Let $p_{i}$ be the point of intersection of $L_{i}$ with $L$. Since $p$ is modular, every line in $\mathcal{L}$ (other than $L$ itself) must intersect $L$ at one of the points $p_{i}$. We ant to show that one of the points $p_{i}$ has multiplicity 2 . Suppose by way of contradiction that the multiplicity of $p_{i}$ is more than 2 for each $i$. Thus we can pick an additional line $H_{i}$ in $\mathcal{L}$ through $p_{i}$ for each $i$. The slope of $H_{i}$ in the affine picture at right in Figure 4 is defined and not 0.

For each $i \neq$, the intersection of $H_{i}$ and $H_{j}$ must be on one of the lines $L_{k}$, since $p$ is modular. If the slopes of $H_{1}$ and $H_{m}$ have the same sign, it is easy to see that they intersect either above $L_{m}$ (if the slopes are both positive and $H_{1}$ has the larger slope, or if the slopes are both negative and $H_{1}$ has the more negative slope) or below $L_{1}$ (if the slopes are both positive and $H_{m}$ has the larger slope, or if the slopes are both negative and $H_{m}$ has the more negative slope).

Thus in order for $p$ to be modular, $H_{1}$ and $H_{m}$ must have slopes of opposite sign. This means as you go from $H_{1}$ to $H_{2}$ and on to $H_{m}$, there is a least $i$ such that $H_{i}$ and $H_{i+1}$ have slopes of opposite sign. But this means that $H_{i}$ and $H_{i+1}$ intersect between $L_{i}$ and $L_{i+1}$ and hence that the point of intersection is not on any of the horizontal lines $L_{k}$, contradicting modularity of $p$. Thus at least one of the points $p_{i}$ must have multiplicity 2. (For example, we could have $p_{m}$ have multiplicity 2 so there would be no $H_{m}$, and $H_{1}, \ldots, H_{m_{1}}$ could all meet at a point of $L_{m}$.)

We now state and give a simplified proof of a slightly strengthened version of [1, Theorem 2.4].

Theorem 15. Let $\mathcal{L}$ be a real non-pencil supersolvable line arrangement containing $s$ lines. Let $p$ be any modular point of $\mathcal{L}$ and let $m$ be the multiplicity of $p$. Then $t_{2} \geq \max \{s-m, m\} \geq s / 2$.

Proof. By Lemma 14, each of the $s-m$ lines in $\mathcal{L}$ not through $p$ contain a point of multiplicity 2. These points are all distinct since if two different lines not through $p$ shared a point of multiplicity 2 , no other lines in $\mathcal{L}$ could contain that point, hence no line through $p$ could contain the point, contradicting modularity of $p$. Thus $t_{2} \geq s-m$. On the other hand, by Inequality (2.3) we have $t_{2} \geq 3+(m-3) t_{m} \geq 3+(m-3)=m$. 

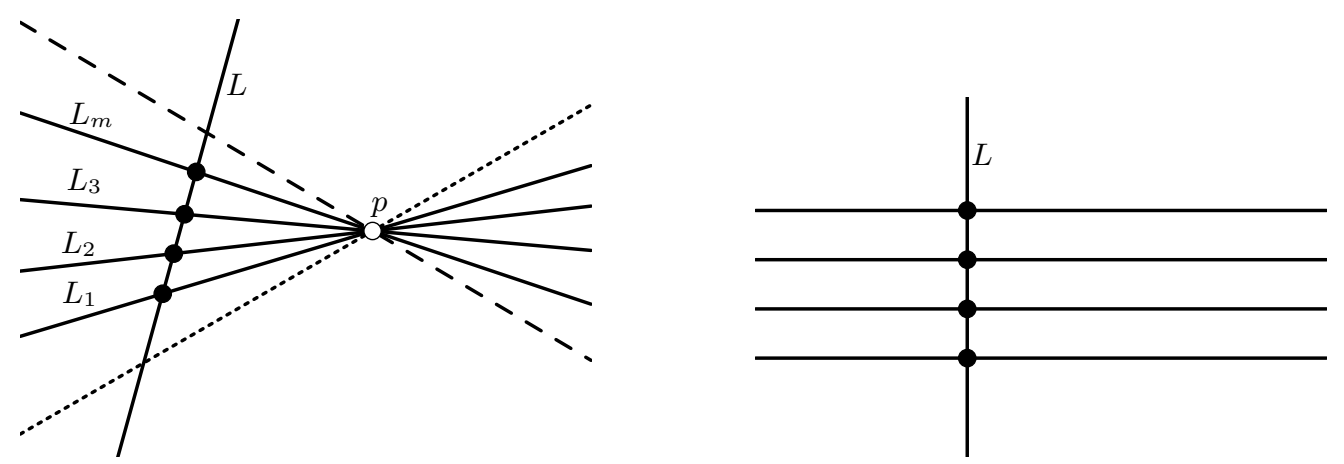

Figure 4. At left, a modular point $p$ of multiplicity $m$ in a real supersolvable line arrangement $\mathcal{L}$ and a line $L$ in $\mathcal{L}=\left\{L_{1}, \ldots, L_{m}\right\}$ not through $p$, and at right an affine version of the same arrangement after an appropriate change of coordinates moving the dashed line to infinity.

The preceding result prompts the following question:

Question 16. Does every non-pencil supersolvable complex line arrangement of s lines with a modular point of multiplicity $m$ satisfy $t_{2} \geq \max \{s-m, m\}$ ?

In the direction of Conjecture 11, we prove the following result.

Theorem 17. Let $\mathcal{L}=\left\{L_{1}, \ldots, L_{s}\right\}$ be a nontrivial complex line arrangement (i.e., not a pencil or near pencil). Assume that every crossing point of $\mathcal{L}$ has multiplicity equal to 3 or 4 . Then the line arrangement $\mathcal{L}$ is not supersolvable.

Proof. Since $\mathcal{L}$ is not a pencil or a near pencil by hypothesis, we can apply Inequality (2.2). In our case, it takes the form: $\frac{3}{4} t_{3} \geq s$.

By (2.1), we have $s(s-1)=6 t_{3}+12 t_{4}$.

Suppose that $\mathcal{L}$ is supersolvable. Then, by (2.4), we have $t_{2} \geq 2 n-m(s-m)-2$, where $n$ is the total number of crossings and $m$ is the maximum $k$ such that $t_{k}>0$. In our case, this gives $0 \geq 2\left(t_{3}+t_{4}\right)-m(s-m)-2$, where $m=3$ or $m=4$.

First we assume $m=4$ and obtain a contradiction. We have $2(s-4)+1 \geq t_{3}+t_{4}$. This implies $12(s-4)+6 \geq 6\left(t_{3}+t_{4}\right) \geq 8 s+6 t_{4}$. The last inequality follows from the Hirzebruch inequality. So we get $6 t_{4}+12(s-4)+6 \geq 6 t_{3}+12 t_{4}=s(s-1)$, where the last equality follows from (2.1).

This, in turn, gives, $12(s-4)+6 \geq 6 t_{4}+8 s \geq s(s-1)-12(s-4)-6+8 s$. Looking at the first and third terms in this and rearranging terms, we get $s^{2}-17 s+84 \leq 0$. But since this quadratic in $s$ has positive leading coefficient and negative discriminant, $s^{2}-17 s+84>0$ for every $s$, giving us the desired contradiction.

The calculation is similar if $m=3$. By (2.4), we get $3(s-3)+2 \geq 2 t_{3}$. Using the Hirzebruch inequality (2.2), we get $9(s-3)+6 \geq 6 t_{3} \geq 8 s$. This forces $s \geq 21$. On the other hand, $s(s-1)=6 t_{3}$ by (2.1). Hence we obtain $9(s-3)+6 \geq 6 t_{3}=s(s-1)$, or equivalently, $(s-3)(s-7) \leq 0$. So $3 \leq s \leq 7$. This is not possible.

Example 18. We do not know many nontrivial examples of complex line arrangements where every crossing point has multiplicity 3 or 4 . We get two examples by taking the lines defined by the linear factors of $\left(x^{n}-y^{n}\right)\left(x^{n}-z^{n}\right)\left(y^{n}-z^{n}\right)$ for $n=3$ and $n=4$. 
The only other example we know is the one due to Klein [10], having 21 lines with $t_{k}=0$ except for $t_{3}=28$ and $t_{4}=21$.

Remark 19. Let $\mathcal{L}$ be a supersolvable line arrangement in $\mathbb{P}_{\mathbb{C}}^{2}$ of $s$ lines. Assume that $t_{2}=0$ and let $m$ be the largest multiplicity of a crossing point of $\mathcal{L}$. By Theorem 17 , we must have $m \geq 5$. Arguing as in the proof of Theorem 17, we can show that, for a fixed $m, s$ must be in a specific set of positive integers. For example, if $m=5$, then our arguments show that $10 \leq s \leq 13$. Similarly, if $m=100$, then $103 \leq s \leq 7412$. But we do not know of any supersolvable line arrangements in $\mathbb{P}_{\mathbb{C}}^{2}$ for which $m=100$ and $s \geq 7413$. If there is such a line arrangement, then our argument shows that it must contain a point of multiplicity 2 .

\section{Applications to unexpectedness}

One of the most interesting applications of line arrangements in $\mathbb{P}^{2}$ is to finding unexpected curves. More specifically, given a line arrangement in $\mathbb{P}^{2}$ one considers the dual arrangement of points. The question then is whether these points admit an unexpected curve. For more details, see [4].

The existence of unexpected curves depends on some properties of the line arrangement. If the arrangement is supersolvable, then [5, Theorem 3.17] proves that there is an unexpected curve through the dual points if and only if $s>2 m$, where $s$ is the number of lines and $m$ is the maximum multiplicity of a crossing point. We now use this characterization to determine which supersolvable arrangements in the classification of Section 3 admit unexpected curves.

5.1. Real line arrangements admitting unexpected curves. First, let us consider a real supersolvable line arrangement $\mathcal{L}$.

If $\mathcal{L}$ has exactly one modular point, then the only arrangement we know which satisfies the condition $s>2 m$ is given by considering a regular $n$-gon for even $n$ and adding the line at infinity. For more details, see [5, Theorem 3.15].

If $\mathcal{L}$ has exactly two modular points, then the only arrangement which admits an unexpected curve is given by the following. Let $m \geq 6$ be even and consider an arrangement of $m$ horizontal and $m$ vertical lines, along with the line at infinity. This is supersolvable with the two modular points of multiplicity $m+1$ at infinity where the horizontal and vertical lines meet the line at infinity. Since there are only $s=2 m+1$ lines, this arrangement does not admit an unexpected curve. But we can add the two diagonals (as in Figure 3, which shows the case of $m=4$, but in that case there are three modular points) to this arrangement without changing the maximum multiplicity while preserving supersolvability. Now the condition $s=2 m+3>2(m+1)$ is satisfied and hence the new arrangement admits an unexpected curve. This arrangement is a special type of tic-tac-toe arrangement described in [5, Theorem 3.19]. The multiplicities of the two modular points (or three when $m=4$ ) in this tic-tac-toe arrangement are equal. There are no other supersolvable arrangements with exactly two modular points which admit unexpected curves.

The only other real supersolvable line arrangement admitting an unexpected curve is the Fermat arrangement for $n=2$ with three coordinate axes added. More precisely, 
this arrangement is defined by $x y z\left(x^{2}-y^{2}\right)\left(x^{2}-z^{2}\right)\left(y^{2}-z^{2}\right)=0$. This has 9 lines and three modular points of multiplicity 4 each (it is displayed in Figure 3).

In summary, except for possibly more supersolvable arrangements with a unique modular point, the only real supersolvable line arrangements which admit an expected curve are listed above. We ask the following question.

Question 20. Are there any other real supersolvable line arrangements (other than the one coming from a regular n-gon) with exactly one modular point whose dual points admit an unexpected curve?

5.2. Complex line arrangements admitting unexpected curves. We now consider complex line arrangements. The only examples known to us of supersolvable arrangements which admit unexpected curves are obtained by adding two or three coordinate axes to the Fermat arrangement $\mathcal{F}_{n}$. In other words, we are considering the complex line arrangement given by $x y\left(x^{n}-y^{n}\right)\left(x^{n}-z^{n}\right)\left(y^{n}-z^{n}\right)$, or $x y z\left(x^{n}-y^{n}\right)\left(x^{n}-z^{n}\right)\left(y^{n}-z^{n}\right)=0$.

This has $s=3 n+\epsilon$ lines, where $\epsilon=2$ or 3 and maximum multiplicity $m=n+2$. Hence the condition $s>2 m$ is satisfied for $\varepsilon=2, n \geq 3$ or $\varepsilon=3, n \geq 2$. In the first case, there is a unique modular point and in the second case, there are three modular points.

We end with the following question.

Question 21. Are there any other complex supersolvable line arrangements (different from the arrangements coming from the Fermat arrangement described above) whose dual points admit an unexpected curve?

\section{REFERENCES}

[1] B. Anzis and Ş. O. Tohăneanu, On the geometry of real or complex supersolvable line arrangements, J. Combin. Theory Ser. A 140 (2016), 76-96.

[2] T. Bauer, S. Di Rocco, B. Harbourne, J. Huizenga, A. Lundman, P. Pokora and T. Szemberg, Bounded negativity and arrangements of lines, Int. Math. Res. Not. IMRN 2015, no. 19, 9456-9471.

[3] T. Bauer, S. Di Rocco, B. Harbourne, J. Huizenga, A. Seceleanu, T. Szemberg, Negative curves on symmetric blowups of the projective plane, resurgences and Waldschmidt constants, International Mathematics Research Notices (2018).

[4] D. Cook II, B. Harbourne, J. Migliore and U. Nagel, Line arrangements and configurations of points with an unexpected geometric property, Compos. Math. 154 (2018), no. 10, 2150-2194.

[5] M. Di Marca, G. Malara, A. Oneto, Unexpected curves arising from special line arrangements, to appear, Journal of Algebraic Combinatorics (arXiv:1804.02730).

[6] G. Dirac, Collinearity properties of sets of points, Quart. J. Math. 2 (1951), 221-227.

[7] M. Dumnicki, B. Harbourne, U. Nagel, A. Seceleanu, T. Szemberg and H. Tutaj-Gasińska, Resurgences for ideals of special point configurations in $\mathbf{P}^{N}$ coming from hyperplane arrangements, $\mathrm{J}$. Algebra 443 (2015), 383-394.

[8] M. Dumnicki, T. Szemberg and H. Tutaj-Gasińska, Counterexamples to the $I^{(3)} \subset I^{2}$ containment, J. Algebra 393 (2013), 24-29.

[9] F. Hirzebruch, Arrangements of lines and algebraic surfaces, in Arithmetic and geometry, Vol. II, 113-140, Progr. Math., 36, Birkhäuser, Boston, MA.

[10] F. Klein, Ueber die Transformation siebenter Ordnung der elliptischen Functionen, Math. Ann. 14 (1878), no. 3, 428-471.

[11] E. Melchior, Über Vielseite der projektiven Ebene, Deutsche Math. 5 (1941), 461-475.

[12] Ş. O. Tohăneanu, A computational criterion for supersolvability of line arrangements, Ars Combin. 117 (2014) 217-223. 
[13] A. Wiman, Zur Theorie der endlichen Gruppen von birationalen Transformationen in der Ebene, Math. Ann. 48 (1896), no. 1-2, 195-240.

Chennai Mathematical Institute, H1 SiPCOT it Park, Siruseri, Kelambakkam 603103, INDIA

E-mail address: krishna@cmi.ac.in

Department of Mathematics, University of Nebraska-Lincoln, Lincoln, Ne 68588, USA

E-mail address: brianharbourne@unl.edu 